\title{
Z-Ligustilide Exerted Hormetic Effect on Growth and Detoxification Enzymes of Spodoptera litura Larvae
}

\author{
Yang Yi, ${ }^{1}$ Guojun Dou, ${ }^{1}$ Zanyang Yu, ${ }^{1}$ Hui He, ${ }^{1}$ Chengqiang Wang, ${ }^{1}$ \\ Li Li, ${ }^{1}$ Jia Zhou, ${ }^{2}$ Dejun Liu, ${ }^{3}$ Jianyou Shi, ${ }^{4}$ Guanrong Li, ${ }^{5}$ Lei Pang, ${ }^{1}$ Na Yang, \\ Qinwan Huang $(\mathbb{D})^{3}$ and Hongyi Qi $\mathbb{D}^{1}$ \\ ${ }^{1}$ College of Pharmaceutical Sciences, Southwest University, Chongqing 400716, China \\ ${ }^{2}$ Sichuan Institute of Food and Drug Control, Chengdu 611731, Sichuan, China \\ ${ }^{3}$ College of Chinese Medicine, Chengdu University of Traditional Chinese Medicine, Chengdu 610075, Sichuan, China \\ ${ }^{4}$ Pharmacy Department, Sichuan Academy of Medical Sciences and Sichuan Provincial People's Hospital, Chengdu 610212, \\ Sichuan, China \\ ${ }^{5}$ College of Agronomy and Biotechnology, Southwest University, Chongqing 400716, China \\ ${ }^{6}$ Institute of Laboratory Animal Sciences, Sichuan Academy of Medical Sciences and Sichuan Provincial People's Hospital, \\ Chengdu 610212, Sichuan, China
}

Correspondence should be addressed to Qinwan Huang; hqwan2163@163.com and Hongyi Qi; hongyiqi@swu.edu.cn

Received 31 January 2018; Revised 3 May 2018; Accepted 2 June 2018; Published 2 July 2018

Academic Editor: Armando Zarrelli

Copyright (C) 2018 Yang Yi et al. This is an open access article distributed under the Creative Commons Attribution License, which permits unrestricted use, distribution, and reproduction in any medium, provided the original work is properly cited.

\begin{abstract}
Plants have evolved a variety of phytochemicals to defense insect feeding, whereas insects have also evolved diverse detoxification enzymes, which are adaptively induced as a prosurvival mechanism. Herein, Z-ligustilide in Ligusticum chuanxiong Hort. was found to exhibit a similar trend in the accumulation from December to May as the occurrence of Spodoptera litura (Fabricius) larvae. Importantly, S. litura larvae feeding enhanced Z-ligustilide level in the stem and leaf $(p<0.01)$. Moreover, Z-ligustilide ranging from 1 to $5 \mathrm{mg} \cdot \mathrm{g}^{-1}$ exhibited remarkable larvicidal activity, antifeedant activity, and growth inhibition against $S$. litura larvae. The $\mathrm{LC}_{50}$ values of larvicidal activity for phthalides in L. chuanxiong were compared as follows: Z-ligustilide > levistilide A > senkyunolide A > 3-butylidenephthalide > senkyunolide I, implicating the critical role of conjugated structure. Notably, there was a biphasic dose response for glutathione S-transferase (GST), cytochrome P450 (CYP) 450, Acetylcholinesterase (AChE), and Carboxylesterase (CarE) activities and GSTs1, cytochrome P450 (CYP) 4S9, and CYP4M14 mRNA expression. Particularly, low dose $\left(0.1 \mathrm{mg} \cdot \mathrm{g}^{-1}\right)$ of Z-ligustilide conferred the resistance of $S$. litura larvae against chlorpyrifos $(p<0.05)$. Together, our data suggest that Z-ligustilide may function in a hormetic way in the chemical defense of $L$. chuanxiong against $S$. litura larvae.
\end{abstract}

\section{Background}

Plants have evolved a variety of phytochemicals that play a chemical defense role against diverse stresses, particularly insect feeding $[1,2]$. It has been well-established that noxious phytochemicals may serve as botanical insecticides due to their good efficacy and environmentally friendly [2]. Importantly, it has also been gradually recognized that phytochemicals produced by plants under stress environment can activate mild cellular stress response mechanisms at a subtoxic level in humans, which may enhance the tolerance against severe dysfunction or disease [3-5], which belongs to a phenomenon of hormesis that is characterized by a biphasic dose response [6]. This is supported by the fact that humans share a great deal of biological similarities to insects, which has established a close coevolutionary relationship with plants over 4 billion years, due to the common ancestor of all multicellular organisms, evolutionary conservation, and adaptive convergence $[7,8]$.

Ligusticum chuanxiong Hort. is a well-known Chinese medicinal and edible plant of the family Apiaceae and mainly produced in Sichuan province [9]. Rhizoma Chuanxiong is 
the dried rootstock of $L$. chuanxiong and traditionally used as an herbal medicine with beneficial effects for stroke, migraine, and gynecological disorders. Moreover, Rhizoma Chuanxiong is frequently stewed with chicken or fish as food therapy. Z-Ligustilide (Z-LIG) is a phthalide compound and accounts for the highest content in the essential oil of $L$. chuanxiong [10]. Recently, we and other investigators found that Z-LIG is one of main components responsible for the neuroprotection of Rhizoma Chuanxiong against ischemic stroke [11-16]. In particular, our investigations demonstrated that pretreatment with Z-LIG remarkably enhanced the tolerance of neuron-like PC12 cells and rats against ischemic injury through activating the cellular stress response pathways Nrf2 and HSP70 [14-16]. Thus, it is possible that Z-LIG belongs to hormetic phytochemicals. In previous screening studies of agrochemicals, Z-LIG exhibited strong insecticidal activity against Drosophila melanogaster [17, 18], mosquito larvae [19], and Bemisia tabaci [20]. However, it is still totally unknown whether Z-LIG plays a defense role in $L$. chuanxiong against diverse stresses, especially, insect feeding.

Spodoptera litura (Fabricius) (Lepidoptera: Noctuidae) is a regular polyphagous and damaging insect and distributed in the tropical and subtropical areas around the world [21, 22]. The wide outbreak of S. litura may be attributed to its migration, high reproductive performance, and detoxification mechanisms $[23,24]$. Most recently, a recombinant protein of $\alpha$-amylase/subtilisin inhibitor cloned from Rhizoma Chuanxiong exhibited potent inhibition against $\alpha$ amylase and subtilisin A from S. litura larvae, indicating the defense mechanisms against $S$. litura existing in $L$. chuanxiong [22]. Curiously, little attention until now has been given to the occurrence of S. litura larvae in L. chuanxiong and the chemical defense role of phytochemicals, such as Z-LIG, in $L$. chuanxiong against $S$. litura larvae.

In the current study, we first determined the potential relationship between the accumulation of Z-LIG and the occurrence of S. litura larvae in L. chuanxiong. Then, the larvicidal activity, antifeedant activity, and growth inhibition of Z-LIG against S. litura larvae were evaluated. Moreover, influence of Z-LIG on the activities and/or the expression of detoxification enzymes in S. litura larvae was further examined. Finally, whether low dose of Z-LIG leads to the resistance of $S$. litura larvae against chlorpyrifos was particularly explored.

\section{Materials and Methods}

2.1. Chemicals. Z-LIG, levistilide A, senkyunolide A, senkyunolide I, and 3-butylidenephthalide were obtained from Chengdu Chroma-Biotechnology Co., Ltd. (Chengdu, China), and stored at $-80^{\circ} \mathrm{C}$ before use. Chlorpyrifos $(\mathrm{O}, \mathrm{O}-$ diethyl O-3,5,6-trichloro-2-pyridyl phosphorothioate, CPF) was purchased from J\&K Scientific Ltd. (Beijing, China).

2.2. Extraction of Essential Oil. The plant material of $L$. chuanxiong was collected and separated as two parts, the rhizome and the stem and leaf. After weighing, the samples were extracted through the hydrodistillation process in a Clevenger Apparatus with continuous ice-cold water circulation in condenser. The volatile fraction was collected in dichloromethane and concentrated by a Buchi rotavapour system with water bath temperature no more than $25 \pm 1^{\circ} \mathrm{C}$.

2.3. Gas Chromatography-Mass Spectrometry (GC-MS). The Z-LIG level in in the essential oils was analyzed by GCMS. The system consists of an Agilent 6890 type gas chromatograph and an Agilent 5975N mass spectrometer, which equips with an Agilent HP-5MS 5\% Phenyl Methyl Siloxane $(30 \mathrm{~m} \times 0.25 \mu \mathrm{m} \times 250 \mu \mathrm{m})$. The analytical conditions are shown as follows: oven and initial temperature $=60^{\circ} \mathrm{C}$ for $3 \mathrm{~min}$, ramp at $8^{\circ} \mathrm{C} / \mathrm{min}$ to $180^{\circ} \mathrm{C}$, then ramp at $5^{\circ} \mathrm{C} / \mathrm{min}$ to $240^{\circ} \mathrm{C}$; injection temperature $=230^{\circ} \mathrm{C}$; injection volume $=1$ $\mu \mathrm{L}$ (1:1000 diluted in acetone); carrier gas $=\mathrm{He}$; flow rate $=1.5$ $\mathrm{mL} / \mathrm{min}$; solvent delay $=0 \mathrm{~min}$; transfer temperature $=250^{\circ} \mathrm{C}$; source temperature $=230^{\circ} \mathrm{C}$; ionization, electron impact; ion energy $=70 \mathrm{eV}$; scan range, 30-500 $\mathrm{Da}$. To quantify the content of Z-LIG, standard compound of Z-LIG with purity more than $98 \%$ was used in GC-MS analysis. Z-LIG was identified based on the retention time and mass spectral comparison with standard compound.

2.4. Insect Rearing. Eggs of S. litura were originally collected from field of $L$. chuanxiong. Then, the rearing was carried out in our laboratory under constant conditions: $27 \pm 1^{\circ} \mathrm{C}, \mathrm{RH} 60$ $70 \%$, and 12L:12D photoperiod. To maintain the generation of insects, female and male moths with the same number were caged in a $10 \mathrm{~cm}$ diameter beaker supplied with honey solution (10\%) and permitted to copulate for $48 \mathrm{~h}$. Eggs were then laid by female moths on wax paper. Upon egg hatching, S. litura larvae were fed on standard artificial diet [25].

2.5. Larvicidal Activity. Test compounds were dissolved in $200 \mu \mathrm{L}$ of DMSO and mixed thoroughly with $100 \mathrm{~g}$ artificial diet. The artificial diet with DMSO only was used as control diet. The artificial diet with different doses of compounds or control diet was placed into Petri dishes $(9 \mathrm{~cm} \times 1.5 \mathrm{~cm})$, respectively. The third instar larvae that already starved for $4 \mathrm{~h}$ were introduced into each Petri dish. After $72 \mathrm{~h}$, the diet was replaced with normal diet. Another 6 days later, mortality of the larvae was checked, and larvae showed that the inability to move was scored as dead. A total of 20 larvae were used in each triplicate. Abbott's formula [26] was applied to calculate the corrected mortality as follows: $P=(T-C) /(100-$ $C) \times 100$, where $P$ refers to $\%$ corrected mortality, $T$ refers to $\%$ death in treatment, and $C$ refers to $\%$ death in vehicle control.

2.6. Antifeedant Activity. Both selective antifeedant activity and nonselective antifeedant activity were evaluated in our study. For the determination of selective antifeedant activity, the test compound-containing artificial diet and the control diet were simultaneously placed into each Petri dish with 3 $\mathrm{cm}$ distance. Twenty of the third instar larvae that already starved for $4 \mathrm{~h}$ were introduced into each Petri dish. The number of larvae within $1 \mathrm{~cm}$ range of the diet was counted within 2 to $72 \mathrm{~h}$ after the introduction of larvae. The selective antifeedant rate $(\%)$ is calculated as $(C-T) /(C+T) \times 100$, where $C$ refers to the average larvae number of the control group and $T$ refers to the average larvae number of the treated group. For 
Table 1: Primers used for semiquantitative RT-PCR.

\begin{tabular}{|c|c|}
\hline Genes & Primer sequences \\
\hline GSTs1 (HQ667936.1) & $\begin{array}{l}\text { F 5'-AACATTGTCGTCATTGGACA-3' } \\
\text { R 5'-ACTTGCTGGTCTCAAAT TTC-3' }\end{array}$ \\
\hline CYP4M14 (DQ352137) & $\begin{array}{l}\text { F5'-GTTGCTCGCTAATCATAGGAAAAT-3' } \\
\text { R } 5^{\prime} \text {-GGTTCTTAAACAAATCTGGTCTCAA-3 }{ }^{\prime}\end{array}$ \\
\hline CYP4S9 (DQ355383) & $\begin{array}{c}\text { F5'-CAACGATGTCTGATCTGGCT-3' } \\
\text { R 5'-GCAGGTCGTATATGTGGATTGAT-3' }\end{array}$ \\
\hline$E F-1 \alpha(\mathrm{U} 20129)$ & $\begin{array}{l}\text { F5'-ATGCCGAAATACGTATTCCACTAC-3' } \\
\text { R 5'-TCCATATTCTAAGCAGGAACTGCA-3' }\end{array}$ \\
\hline
\end{tabular}

F: forward primer; R: reverse primer.

the determination of nonselective antifeedant activity, one piece of the test compound-containing artificial diet or the control diet was placed into a Petri dish. Ten of the third instar larvae that already starved for $4 \mathrm{~h}$ were introduced into each Petri dish. After $48 \mathrm{~h}$, the nonselective antifeedant rate (\%) is calculated as $(C-T) / C \times 100$, where $C$ refers to the average diet weight of the control group before and after the bioassay and $T$ refers to the average diet weight of the treated group before and after the bioassay.

2.7. Growth Inhibition. One piece of the test compoundcontaining artificial diet or the control diet was placed into a $5 \mathrm{~cm}$ diameter beaker. Ten of the third instar larvae were introduced into each beaker. After $48 \mathrm{~h}$, the diet was replaced with normal diet. The larvae were continuously reared to become pupae. The average weight of pupae was then calculated.

2.8. Determination of Glutathione-S-Transferase (GST), Cytochrome P450 (CYP) CYP450, Acetylcholinesterase (AChE), and Carboxylesterase (CarE) Activities. The fifth instar larvae were exposed to the test compound-containing artificial diet or the control diet for $48 \mathrm{~h}$. Midguts separated from larvae of each treatment were homogenized in liquid nitrogen and then added with 5 volumes of $0.1 \mathrm{M}$ phosphate buffer $(\mathrm{pH}$ 7.0). Each sample was centrifugated at $8000 \mathrm{~g}$ for $10 \mathrm{~min}$ at $4^{\circ} \mathrm{C}$. Then, the activities of GST, AChE, CYP450, and CarE were further determined with the respective commercial kits (Nanjing Jiancheng Biotechnology, China) following the instructions provided by manufacturer.

2.9. Semiquantitative RT-PCR Detection of GSTs1, Cytochrome P450 (CYP) 4M14, and CYP4S9. The fifth instar larvae were exposed to the test compound-containing artificial diet or the control diet for $48 \mathrm{~h}$. Total RNA was isolated from midguts of S. litura larvae by using TRIzol ${ }^{\circledR}$ reagent (Invitrogen) and liquid nitrogen. The RT-PCR was performed with PrimeScript ${ }^{\mathrm{TM}}$ RT reagent Kit and Premix Taq (Takara Bio USA, Inc., USA) following the instructions provided by the manufacturer. The primer was designed according to the sequence of each gene in GeneBank and the specific primer sequences for GSTs1 (HQ667936.1), CYP4M14 (DQ352137), CYP4S9 (DQ355383), and Elongation factor-1 $\alpha,(E F-1 \alpha)$ (U20129) were shown in Table 1[27, 28]. EF-1 $\alpha$ was used as internal control. PCR amplification was set as follows: after an initial denaturation at $94^{\circ} \mathrm{C}$ for $3 \mathrm{~min}, 32$ cycles of $94^{\circ} \mathrm{C}$ for $30 \mathrm{~s}, 55.9^{\circ} \mathrm{C}$ for $45 \mathrm{~s}$, and $72^{\circ} \mathrm{C}$ for $60 \mathrm{~s}$. The reaction ended with a final extension step at $72^{\circ} \mathrm{C}$ for $10 \mathrm{~min}$. PCR products were resolved by gel electrophoresis in $1.2 \%$ agarose containing Tanon ${ }^{\circledR}$ Nucleic acid dye and visualized under UV light.

2.10. Statistical Analysis. The data in this study were reported as means $\pm \mathrm{SD}$ for triplicate. The $\mathrm{LC}_{50}$ values were evaluated using Probit analysis. A t-test or one-way ANOVA was applied to evaluate for significant differences between two groups. A $p$-value of less than 0.05 was considered to be statistically significant.

\section{Results}

3.1. Influence of S. litura Larvae Feeding on the Accumulation of Z-LIG in L. chuanxiong. In field observation of L. chuanxiong in the region of Pengzhou in Sichuan province, S. litura larvae were found to be one of the main pests (Figure 1(a)). To determine the incidence of $S$. litura larvae, we evaluated the amount of $S$. litura larvae in L. chuanxiong field during the different growth periods. As shown in Figure 1(b), no S. litura larvae were found during December to February. Only a few S. litura larvae (5 per 100 plants in average) were observed in March. However, the amount of S. litura larvae dramatically increased in part of L. chuanxiong field during April (28 larvae per 100 plants in average) and May (51 larvae per 100 plants in average). Moreover, S. litura larvae were found in almost $62 \%$ of $L$. chuanxiong field. Meanwhile, we also determined the accumulation of Z-LIG in L. chuanxiong by collecting L. chuanxiong from December to May and detecting the content of Z-LIG in the rhizome and the stem and leaf with GC-MS. As shown in Figure 1(c), the level of Z-LIG exhibited little change in rhizome from December to February. Obvious increase of Z-LIG in the rhizome was shown in March. A rapid accumulation of ZLIG in the rhizome was observed in April and May, with 6.77fold and 9.03-fold increase compared with that of December, respectively. The variation trend of Z-LIG content in the stem and leaf is very similar to that in the rhizome. However, the level of Z-LIG in the rhizome is marked higher than that in the stem and leaf, with 6.98-fold and 6.36-fold higher in April and May, respectively. Then, we determined the influence of S. litura larvae feeding on the content of Z-LIG in the potted L. chuanxiong. As shown in Figure 1(d), the Z-LIG 


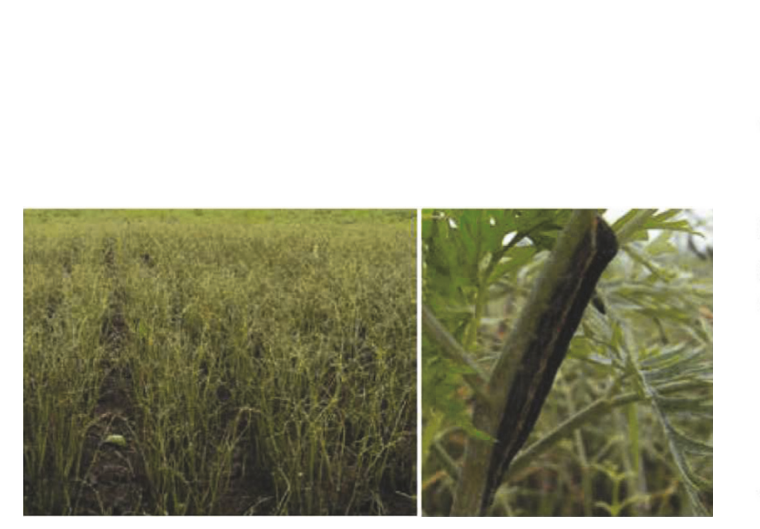

(a)

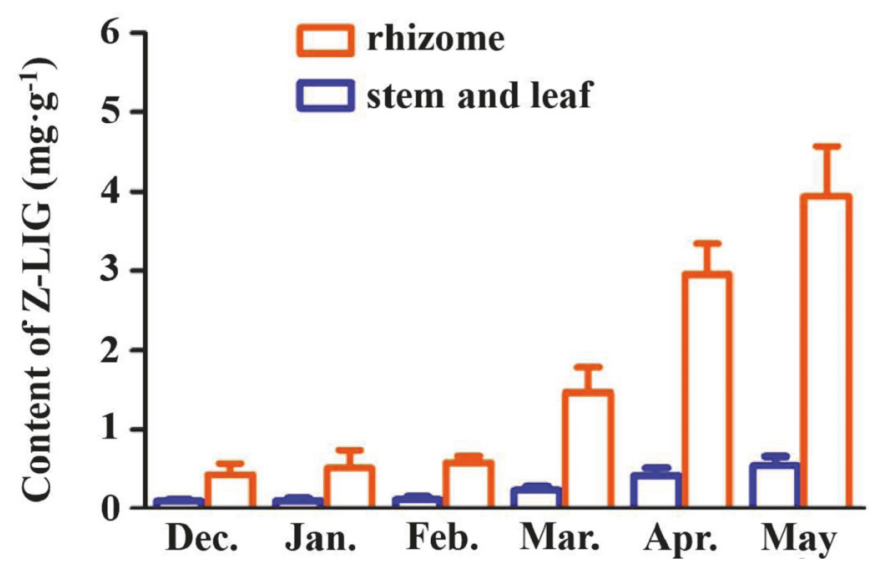

(c)

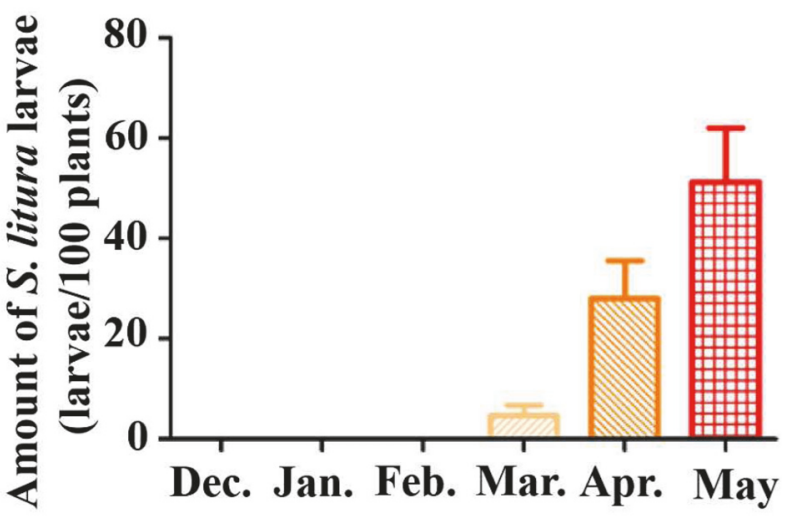

(b)

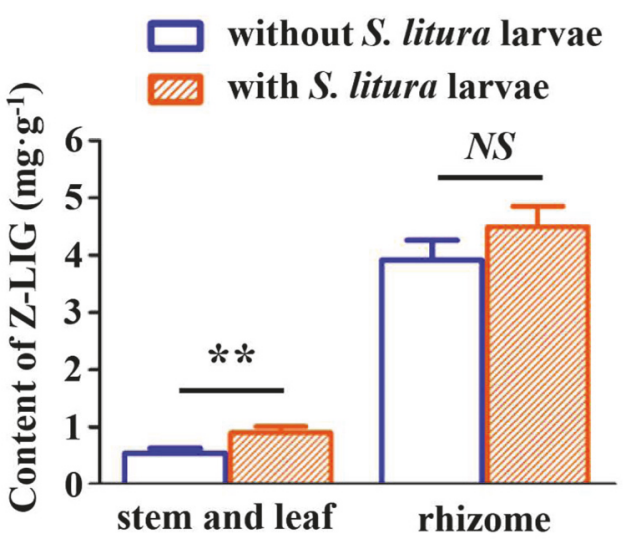

(d)

FIGURE 1: S. litura larvae feeding contributes to the accumulation of Z-LIG in L. chuanxiong. (a) Representative images showing the occurrence of S. litura larvae in L. chuanxiong in the region of Pengzhou in Sichuan province, China. (b) The incidence rate of $S$. litura larvae in $L$. chuanxiong field from December to May. (c) The accumulation of Z-LIG in L. chuanxiong during different growth periods. The Z-LIG content in the rhizome and the stem and leaf was analyzed by GC-MS. (d) Influence of S. litura larvae feeding on the content of Z-LIG in the stem and leaf and the rhizome of the potted L. chuanxiong. After starvation for $4 \mathrm{~h}$, Third instar larvae of $S$. litura were released to feed on the potted L. chuanxiong (2 larvae per plants). After $24 \mathrm{~h}$, the plant materials were collected and the Z-LIG content in the rhizome and the stem and leaf was analyzed by GC-MS. Values are presented as means \pm SD. $(* *) p<0.01$. NS, nonsignificant.

level in the stem and leaf of the potted L. chuanxiong. with $S$. litura larvae feeding (2 larvae per plants for $24 \mathrm{~h}$ ) remarkably increased compared with that without $S$. litura larvae feeding $(p<0.01)$, while the Z-LIG level in the rhizome of the potted $L$. chuanxiong with $S$. litura larvae feeding exhibited an increasing trend, but it is not statistically significant $(p>$ 0.05).

3.2. Larvicidal Activity, Antifeedant Activity, and Growth Inhibition of Z-LIG. To determine the larvicidal activity of Z-LIG on $S$. litura larvae, we fed S. litura larvae with food containing different doses of Z-LIG (0.1 to $\left.5 \mathrm{mg} \cdot \mathrm{g}^{-1}\right)$. As shown in Figure 2(a), 0.1 and $0.25 \mathrm{mg} \cdot \mathrm{g}^{-1}$ of Z-LIG showed almost no insecticidal activity compared with vehicle. Moreover, 0.5 $\mathrm{mg} \cdot \mathrm{g}^{-1}$ of Z-LIG showed only weak larvicidal activity. However, Z-LIG (1 to $5 \mathrm{mg} \cdot \mathrm{g}^{-1}$ ) exhibited prominent larvicidal activity. Then, we determined the antifeedant activity of ZLIG on S. litura larvae. The result of selective antifeedant activity in Figure 2(b) showed that S. litura larvae exhibited even higher selectivity to food with $0.1 \mathrm{mg} \cdot \mathrm{g}^{-1} \mathrm{Z}-\mathrm{LIG}$ than that with vehicle. Very weak selective antifeedant activity was observed for $0.25 \mathrm{mg} \cdot \mathrm{g}^{-1}$ and $0.5 \mathrm{mg} \cdot \mathrm{g}^{-1}$ of Z-LIG, whereas the selective antifeedant rate dramatically increased in $S$. litura larvae exposed to 1 to $5 \mathrm{mg} \cdot \mathrm{g}^{-1}$ of Z-LIG. Moreover, $S$. litura larvae exposed to $5 \mathrm{mg} \cdot \mathrm{g}^{-1}$ of Z-LIG exhibited $100 \%$ of selective antifeedant rate. Figure 2(c) showed that only weak nonselective antifeedant rate was shown in S. litura larvae exposed to 0.1 to $0.5 \mathrm{mg} \cdot \mathrm{g}^{-1}$ of Z-LIG. However, marked selective antifeedant rate was observed in S. litura larvae exposed to 1 to $5 \mathrm{mg} \cdot \mathrm{g}^{-1}$ of Z-LIG. To further examine the effect of Z-LIG on the growth of S. litura larvae, we compared the weight of $S$. litura larvae fed with and without Z-LIG.

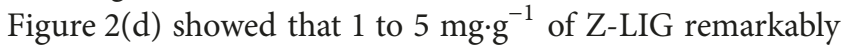
inhibited the weight of $S$. litura larvae and 0.25 to $0.5 \mathrm{mg} \cdot \mathrm{g}^{-1}$ of Z-LIG only exhibited the weak or moderate inhibition, 


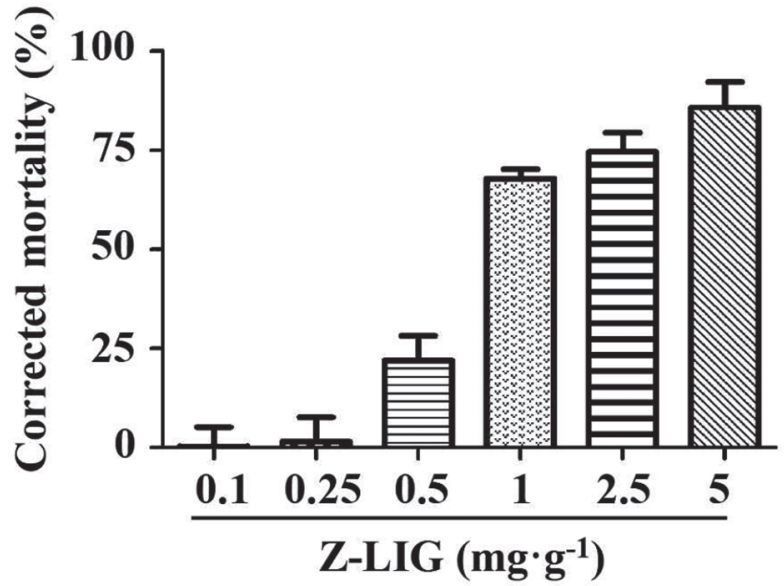

(a)

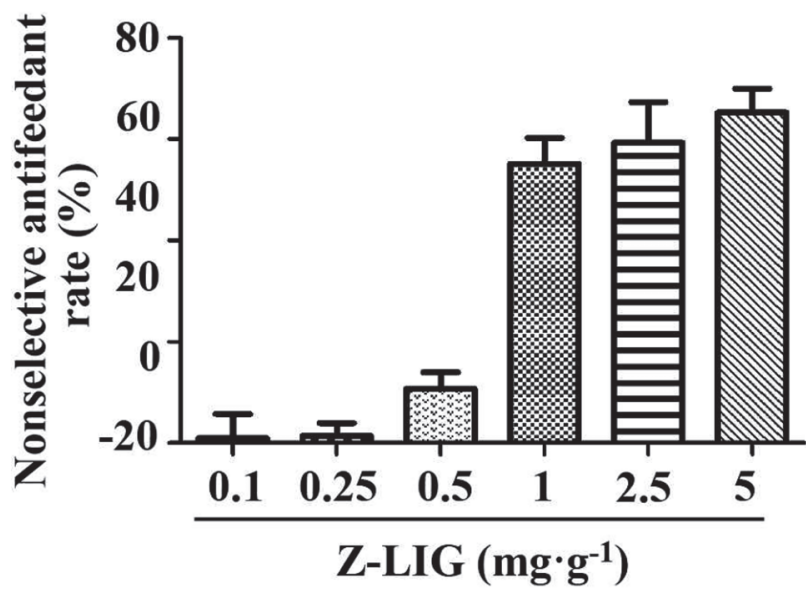

(c)

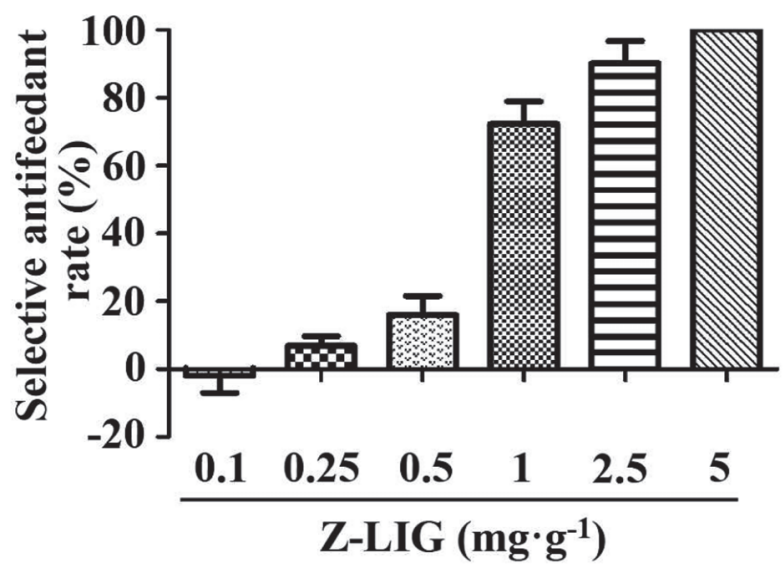

(b)

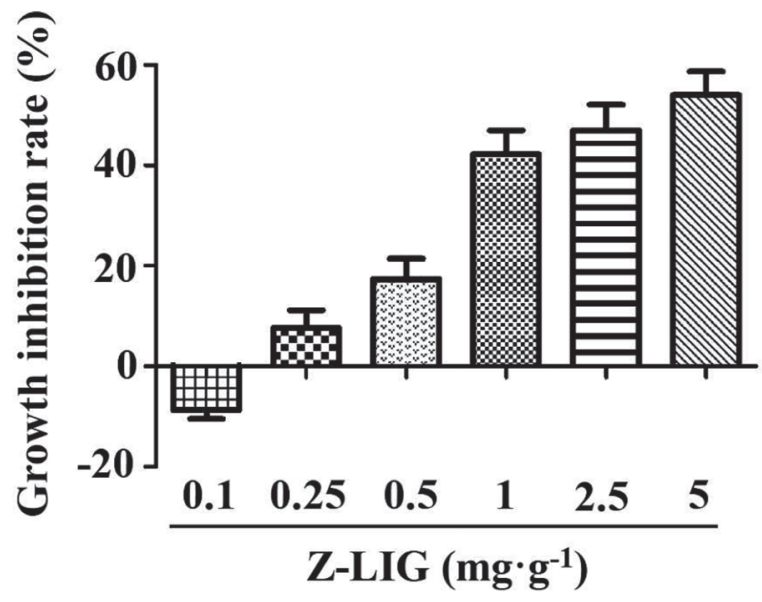

(d)

Figure 2: Defense effect of Z-LIG against S. litura larvae: (a) larvicidal activity; (b) nonselective antifeedant rate; (c) selective antifeedant rate; and (d) growth inhibition. Values are presented as means \pm SD.

whereas $0.1 \mathrm{mg} \cdot \mathrm{g}^{-1}$ of Z-LIG obviously promoted the growth of $S$. litura larvae.

3.3. Larvicidal Activity of Phthalides Existing in the Essential Oil of L. chuanxiong. The larvicidal activity of the five phthalides (Figure 3) existing in the essential oil of L. chuanxiong is shown in Table 2. Z-LIG showed the most larvicidal activity among these phthalides against $S$. litura larvae, with an $\mathrm{LC}_{50}$ value of $0.59 \mathrm{mg} \cdot \mathrm{g}^{-1}$. Levistilide $A$ and senkyunolide A showed the moderate larvicidal activity, with $\mathrm{LC}_{50}$ values of 0.67 and $0.98 \mathrm{mg} \cdot \mathrm{g}^{-1}$, respectively. However, both 3butylidenephthalide and senkyunolide I were less active and exhibited $\mathrm{LC}_{50}$ values of 1.56 and $2.39 \mathrm{mg} \cdot \mathrm{g}^{-1}$, respectively.

3.4. Effect of Z-LIG on GST, CYP450, AChE, and CarE Activities. As shown in Figure 4(a), there was a significant increase of GST activity in S. litura larvae exposed to 0.1 ( $p$ $<0.01)$ and $0.5(p<0.05) \mathrm{mg} \cdot \mathrm{g}^{-1}$ of Z-LIG compared with that exposed to vehicle, whereas GST activity dramatically decreased in $S$. litura larvae exposed to 1 and $5 \mathrm{mg} \cdot \mathrm{g}^{-1}$ of $\mathrm{Z}$ LIG ( $p<0.001)$. Almost 21-fold decrease was observed in $S$. litura larvae exposed to $5 \mathrm{mg} \cdot \mathrm{g}^{-1}$ of Z-LIG compared with that exposed to vehicle. In Figures 4(b) and 4(c), CYP450 and AChE activities also exhibited a similar trend that the activities of both enzymes remarkably increased for 0.1 and $0.5 \mathrm{mg} \cdot \mathrm{g}^{-1}$ of Z-LIG and significantly decreased for 1 and 5 $\mathrm{mg} \cdot \mathrm{g}^{-1}$ of Z-LIG. For CarE activity, a marked increase was observed in $S$. litura larvae exposed to 0.1 to $1 \mathrm{mg} \cdot \mathrm{g}^{-1}$ of $\mathrm{Z}$ LIG compared with that exposed to vehicle and the highest enzyme activity was shown for $0.5 \mathrm{mg} \cdot \mathrm{g}^{-1}$ of Z-LIG.

3.5. Effect of Z-LIG on GSTs1, CYP4S9, and CYP4M14 Expression. To further determine the influence of Z-LIG on the expression of detoxification enzymes, we used RT-PCR to evaluate the mRNA level of GSTs1, CYP4S9, and CYP4M14. Interestingly, the mRNA expression of all these three genes exhibited typic biphasic dose responses after S. litura larvae were exposed to Z-LIG ( 0.1 to $5 \mathrm{mg} \cdot \mathrm{g}^{-1}$ ) or vehicle (Figure 5). The maximum expression for both GSTs1 and CYP4S9 was 
TABLE 2: $\mathrm{LD}_{50}$ values for larvicidal activity of phthalides against $S$. Litura larvae.

\begin{tabular}{|c|c|c|c|c|}
\hline Compound & $\mathrm{LD}_{50}{ }^{a}\left(\mathrm{mg} \cdot \mathrm{g}^{-1}\right)$ & $95 \% \mathrm{CL}^{b}\left(\mathrm{mg} \cdot \mathrm{g}^{-1}\right)$ & slope \pm SE & $\mathrm{R}^{2}$ \\
\hline$\overline{Z \text { Z-LIG }}$ & 0.59 & $0.51-0.69$ & $1.03 \pm 0.28$ & 0.9594 \\
\hline senkyunolide A & 0.98 & $0.84-1.13$ & $0.86 \pm 0.20$ & 0.9649 \\
\hline senkyunolide I & 2.39 & $2.04-2.80$ & $0.91 \pm 0.23$ & 0.9611 \\
\hline levistilide A & 0.67 & $0.58-0.76$ & $0.93 \pm 0.21$ & 0.9688 \\
\hline 3-butylidenephthalide & 1.56 & $1.35-1.81$ & $0.88 \pm 1.21$ & 0.9657 \\
\hline
\end{tabular}

${ }^{\mathrm{a}} \mathrm{LC}_{50}$ is the lethal concentration for $50 \%$ mortality; ${ }^{\mathrm{b}} 95 \%$ confidence limit.<smiles>CCCC=C1OC(=O)C2C=CCCC12</smiles>

Z-ligustilide<smiles>CCC/C=C1\OC(=O)C2=C1CC[C@@H]1[C@@H]3C=C4C(=O)O/C(=C\CCC)[C@]4(C3)[C@H]21</smiles>

levistilide A<smiles>CCCC[C@H]1OC(=O)C2=C1CCC=C2</smiles><smiles>CCCC=C1OC(=O)c2ccccc21</smiles><smiles>CCC/C=C1\OC(=O)C2=C1CC[C@H](O)[C@H]2O</smiles>

\section{senkyunolide A 3-butylidenephthalide senkyunolide I}

FIGURE 3: Structure of main phthalides existing in L. chuanxiong.

observed in $S$. litura larvae exposed to $0.1 \mathrm{mg} \cdot \mathrm{g}^{-1}$ of Z-LIG, while $0.5 \mathrm{mg} \cdot \mathrm{g}^{-1}$ of Z-LIG caused maximum expression of CYP4M14.

3.6. Low Dose of Z-LIG Enhanced Resistance of S. litura Larvae against Pesticide CPF. To determine the possible influence of low dose of Z-LIG on the resistance of S. litura larvae, we pretreated $S$. litura larvae with $0.1 \mathrm{mg} \cdot \mathrm{g}^{-1}$ of Z-LIG or vehicle for $48 \mathrm{~h}$ and then treated $S$. litura larvae with $\mathrm{CPF}$ $\left(2.5 \mu \mathrm{g} \cdot \mathrm{g}^{-1}\right)$ or vehicle for another 3 days and the survival rate was determined. As shown in Figure 6, there was no S. litura larvae death in the group pretreated and treated with vehicle. The same result was also observed in the group pretreated with Z-LIG and treated with vehicle. However, there was only $46.67 \%$ survival rate in the group of vehicle pretreatment and CPF treatment. Notably, the survival rate significantly increased to $60 \%$ in the group of Z-LIG pretreatment and $\mathrm{CPF}$ treatment $(p<0.05)$. These results suggest that low dose of Z-LIG pretreatment may enhance the resistance of S. litura larvae against $\mathrm{CPF}$.

\section{Discussion}

In an ecological environment, plants have evolved sophisticated defense systems to sense and resist a variety of biotic threats. Invertebrates, particularly insects, are the major biotic factor that interacts with plants in the microenvironment as insects have evolved to feed on plants at least 4 billion years ago, which leads to the formation of a close coevolutionary relationship [29]. The main approach employed by plants to avoid the attack from herbivorous insects is the chemical defense, which is partially mediated by the production of toxic and repellent compounds that accumulate after insect feeding [30, 31]. Recently, we directed our effort to screen hormetic phytochemicals from essential oils extracted from traditional Chinese herbal medicine. As a result, a group of phthalide compounds isolated from the essential oil of Rhizoma Chuanxiong drew our special attention. Among them, Z-LIG exhibited potent neuroprotective effect against ischemic injury via the activation of cellular stress response mechanism Nrf2 and HSP70 pathways [14-16]. Meanwhile, the role that Z-LIG plays in L. chuanxiong is also very interesting to us. Rhizoma Chuanxiong collected from the regions of Dujiangyan and Pengzhou in Sichuan province is of high quality and large quantities and supplies nationwide and abroad [9]. It has been found that S. litura larvae are one of the major pests that feed on L. chuanxiong distributed in Dujiangyan and Pengzhou [32]. Thus, the current study aimed to figure out whether Z-LIG plays a defense role in $L$. chuanxiong against $S$. litura larvae. 


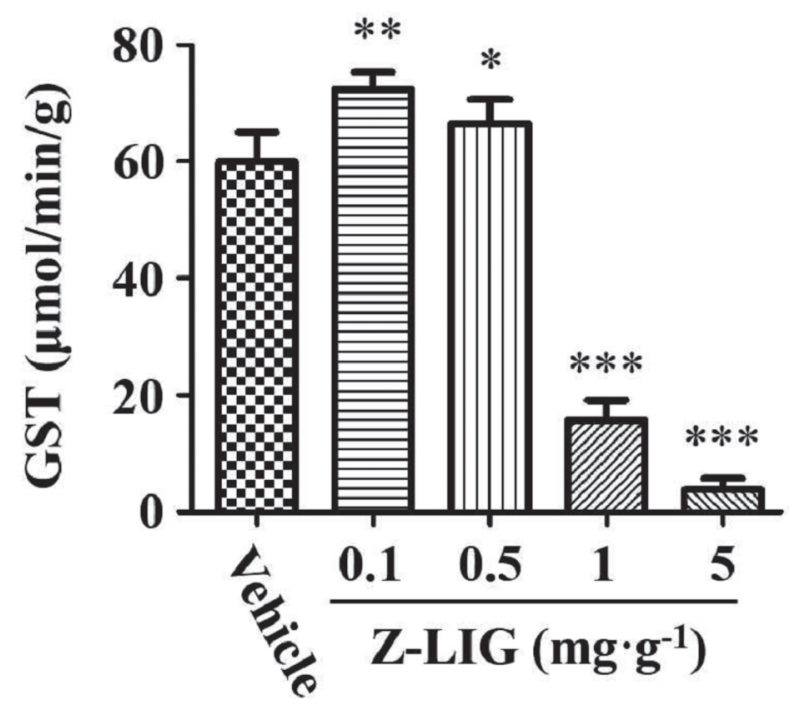

(a)

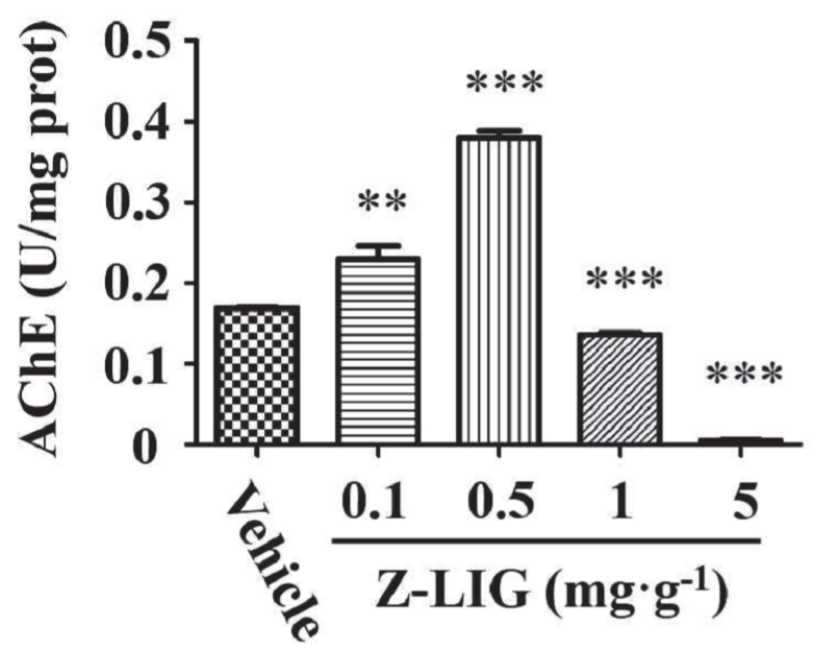

(c)

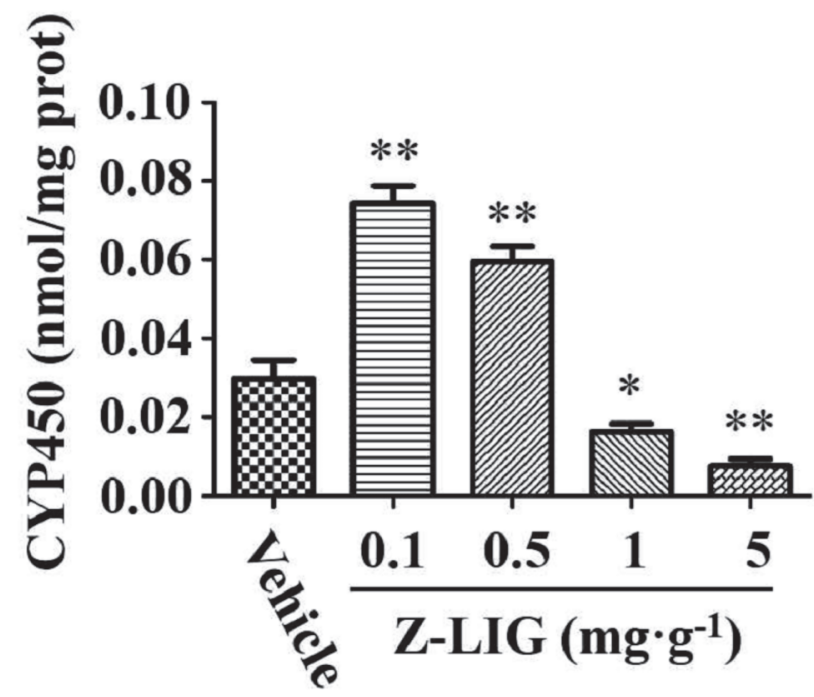

(b)

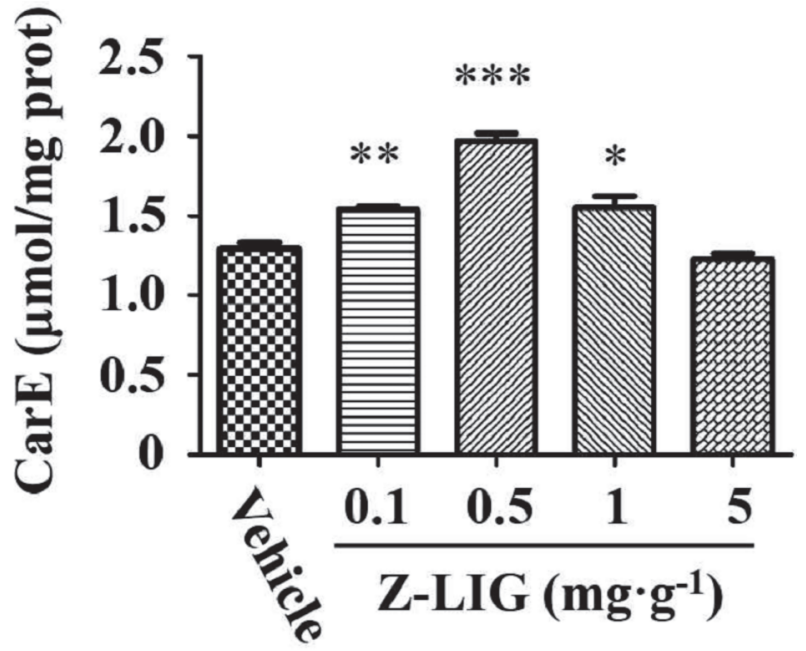

(d)

FIGURE 4: Effect of Z-LIG on the activities of detoxification enzymes GST, AChE, CYP450, and CarE. Enzyme activities were determined as mentioned under Materials and Methods. Values are presented as means \pm SD. $(*) p<0.05,(* *) p<0.01$, and $(* * *) p<0.001$.

In the field of $L$. chuanxiong in Pengzhou of Sichuan province, we found that $S$. litura larvae were indeed one of the main pests of $L$. chuanxiong. Thus, we first determined the incidence of $S$. litura larvae in L. chuanxiong and the accumulation of Z-LIG in the rhizome and the stem and leaf during the different periods of growth. Interestingly, we observed that there was a quite similar increasing trend for Z-LIG content and S. litura larvae amount, both of which showed a rapid growth during March to May. Then, we asked whether feeding by S. litura larvae influences the accumulation of Z-LIG in L. chuanxiong. Interestingly, our results showed that $S$. litura larvae feeding led to the increase of Z-LIG in the stem and leaf of the potted L. chuanxiong, suggesting that Z-LIG accumulation in L. chuanxiong may be at least in part correlated with the outbreak of $S$. litura larvae. Similarly, accumulating evidence have demonstrated that phytochemicals are adaptively induced and accumulate in plants in response to attack by insects. For example, the induced accumulation of isoflavone 7-O-glucosides and isoflavone $7-\mathrm{O}-\left(6^{\prime \prime}-\mathrm{O}\right.$-malonyl- $\beta$-glucosides $)$ was observed after soybean (Var. Enrei) leaves were treated with a combination of plant defense elicitors present in S. litura gut content extracts [33]. Two isoflavone aglycones (daidzein and formononetin) and their conjugates (glucosides and malonyl glucosides) were found to increase in soybean leaves [Glycine max (L.) Merr. (Fabaceae)] following attack by common cutworm larvae [34]. The highly toxic isothiocyanates were produced by the myrosinase mediated hydrolysis of glucosinolates in cruciferous plants upon the damage of herbivores [35]. We then evaluated whether Z-LIG showed a defense effect against $S$. litura larvae by determining larvicidal activity, antifeedant activity, and growth inhibition. Our results 


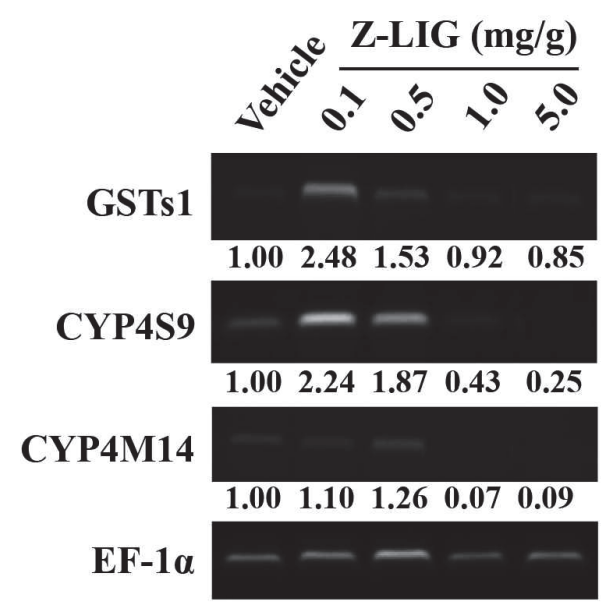

FIGURE 5: Effect of Z-LIG on the mRNA expression of GSTS1, CYP4S9, and CYP4M14. mRNA expression was determined as mentioned under Materials and Methods.

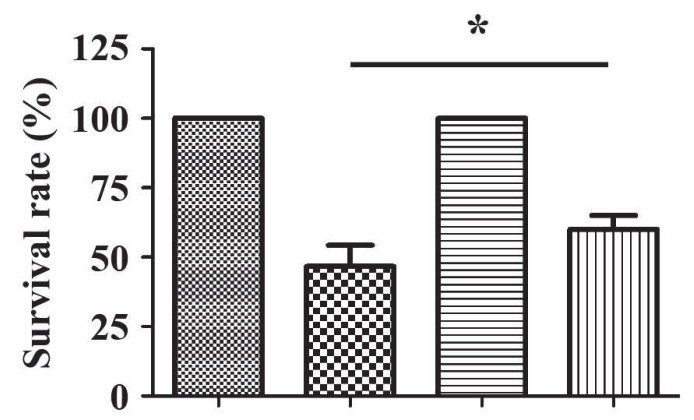

\section{Pre-treatment: DMSO DMSO Z-LIG Z-LIG Treatment: DMSO CPF DMSO CPF}

FIgUre 6: Low dose of Z-LIG confers resistance of $S$. litura larvae against CPF. S. litura larvae were pretreated with $0.1 \mathrm{mg} \cdot \mathrm{g}^{-1}$ of Z-LIG or vehicle for $48 \mathrm{~h}$ and then treated with CPF $\left(2.5 \mu \mathrm{g} \cdot \mathrm{g}^{-1}\right)$ or vehicle for another 3 days and the survival rate was determined. Values are presented as means $\pm \mathrm{SD} ;(*) p<0.05$.

showed that Z-LIG ranging from 0.1 to $0.5 \mathrm{mg} \cdot \mathrm{g}^{-1}$ showed no or weak larvicidal activity, antifeedant activity, and growth inhibitory activity against $S$. litura larvae, whereas higher dose (1 to $5 \mathrm{mg} \cdot \mathrm{g}^{-1}$ ) of Z-LIG exhibited remarkable larvicidal activity, antifeedant activity, and growth inhibitory activity. Previous studies demonstrated that Z-LIG also exhibited strong insecticidal activity against other insects. For example, Z-LIG was shown to be the most potent toxicity among the alkylphthalides isolated from the chloroform extract of Cnidium officinale rhizomes against the larvae and adults of Drosophila melanogaster $[17,18]$ and the Q-biotype females of Bemisia tabaci [20]. Besides Z-LIG, we also determined the larvicidal activity of other structure-similar phthalides existing in the essential oil of L. chuanxiong. Based on $\mathrm{LC}_{50}$ values, the potency of the larvicidal activity of these phthalides against $S$. litura larvae can be summarized as follows: Z-Ligustilide > levistilide A > senkyunolide A > 3butylidenephthalide $>$ senkyunolide I. The structure-activity relationship can be further drawn based on the results. ZLIG bears a typical $\alpha, \beta, \gamma, \delta$-unsaturated lactone. Moreover, the carbonyl group at its C-2 position is an electronwithdrawing group, which can withdraw electron from the $\alpha, \beta, \gamma, \delta$-unsaturated double bonds. Thus, the unsaturated double bond between C-3 and C-8 in Z-LIG is highly active. Levistilide $\mathrm{A}$ is polymerized by two molecular Z-LIG and only one complete chain of $\alpha, \beta, \gamma, \delta$-unsaturated double bonds is preserved. Senkyunolide A lacks $\gamma, \delta$-unsaturated double bond compared with Z-LIG. The aromatic ring of 3-butylidenephthalide is a strong electron donating group, which greatly reduces the reactive ability of the $\gamma, \delta$ unsaturated double bonds. The hydroxyl group at the C7 position of senkyunolide I can form a conjugated effect with $\alpha, \beta, \gamma, \delta$-two double bonds. Under this effect, hydroxyl group is an electron donating group, which also reduces the reactive ability of the $\gamma, \delta$-unsaturated double bonds. Thus, our results indicate the presence of conjugation of $\alpha, \beta, \gamma, \delta$ unsaturated double bonds with carbonyl group rather than aromaticity or hydroxyl group seems to play a critical role for the larvicidal activity of phthalides against $S$. litura larvae. Consistent with our results, Chae et al. [20] reported that the conjugation is highly correlated with the toxicity of three phthalides identified in Rhizome Cnidium officinale (Japanese Chuanxiong) to the Q-biotype females. However, it has also been shown that the conjugation and aromaticity seem to play an important role in the larvicidal and adulticidal activity of phthalides against Drosophila melanogaster $[17,18]$.

Notably, the effect of Z-LIG on the enzyme activities of GST, CYP450, AChE, and CarE in S. litura larvae showed a biphasic dose response with an increase in low dose of Z-LIG and decrease in high dose of Z-LIG, which is supported by Z-LIG-mediated change of GSTS1 mRNA expression, which also exhibited a biphasic dose response. Similar trend was further observed in the mRNA expression of CYP4S9 and CYP4M14 in S. litura larvae after Z-LIG treatment. These results indicate the possibility that detoxification enzymes can be adaptively induced in S. litura larvae as a prosurvival mechanism in response to low dose of Z-LIG, whereas they were directly inhibited under the insult of high dose of $Z$ LIG, which further confirms the hormetic role of Z-LIG. Our previous studies showed that low dose of Z-LIG induced transcription factor Nrf2-mediated detoxification enzymes and HSP70 pathways, which resulted in the tolerance against ischemic injury [14-16]. We are then curious about whether low dose of Z-LIG enhances the resistance of S. litura larvae as we already showed that low dose of Z-LIG induced the expression and/or activity of detoxification enzymes in Figures 4 and 5. Interestingly, our results clearly demonstrated that the resistance of $S$. litura larvae against pesticide $\mathrm{CPF}$ was remarkably enhanced by the pretreatment of low dose $(0.1$ $\mathrm{mg} \cdot \mathrm{g}^{-1}$ ) of Z-LIG. This result obtained from the level of plant and insect interaction may provide an explanation for our recent study, in which subtoxic dose of Z-LIG activated the cellular stress response mechanisms, such as Nrf2 and HSP70 mediated pathways, in rats, and enhanced the tolerance of the rats against ischemic injury [14-16]. 
Under field conditions, S. litura larvae feed mainly on leaf and occasionally on stem of L chuanxiong. In our study, we found that Z-LIG level in the stem and leaf of $L$. chuanxiong was $0.42 \pm 0.10 \mathrm{mg} \cdot \mathrm{g}^{-1}$ in April and $0.55 \pm 0.11 \mathrm{mg} \cdot \mathrm{g}^{-1}$ in May. Under our experimental conditions, Z-LIG ranging from 0.1 to $0.5 \mathrm{mg} \cdot \mathrm{g}^{-1}$ showed weak larvicidal activity, antifeedant activity, and growth inhibitory activity against $S$. litura larvae. Meanwhile, the detoxification enzymes in the midgut of $S$. litura larvae were also adaptively triggered by Z-LIG ranging from 0.1 to $0.5 \mathrm{mg} \cdot \mathrm{g}^{-1}$. Thus, it can be reasonably speculated that Z-LIG produced in the stem and leaf of L. chuanxiong may play a weak chemical defense role against $S$. litura larvae, while $S$. litura larvae also utilize detoxification enzymes as prosurvival mechanism against the toxicity of Z-LIG, which implicating an interaction between $L$. chuanxiong and $S$. litura larvae.

\section{Conclusion}

Our study demonstrated that high dose of Z-LIG exhibited remarkable larvicidal activity, antifeedant activity, and growth inhibition against $S$. litura larvae and dramatically inhibited the activity and/or expression of detoxification enzymes in S. litura larvae. However, low dose of Z-LIG relevant to the level of Z-LIG in L. chuanxiong showed a weak defense effect on S. litura larvae, which was accompanied by the adaptive induction of detoxification enzymes in $S$. litura larvae. These results suggest that Z-LIG may function as a hormetic phytochemical in the chemical defense of $L$. chuanxiong against $S$. litura larvae, which may facilitate our understanding of health benefits of Z-LIG at subtoxic dose in humans from ecological and evolutionary perspective.

\section{Abbreviations}

CYP: Cytochrome P450

CPF: Chlorpyrifos

GST: Glutathione S-transferase

GC-MS: Gas chromatography-mass spectrometry

RT-PCR: Reverse transcriptase-polymerase chain reaction

Z-LIG: Z-ligustilide.

\section{Data Availability}

Access to the data used to support the findings of this study can be considered by the author upon request, with permission of institutional review board. Please contact the corresponding author for the requests.

\section{Conflicts of Interest}

The authors declare no competing financial interest.

\section{Authors' Contributions}

Hongyi Qi and Qinwan Huang conceived and designed the experiment; Yang Yi, Guojun Dou, Zanyang Yu, Hui $\mathrm{He}$, Chengqiang Wang, $\mathrm{Li} \mathrm{Li}$, and Jia Zhou collected the data; Guojun Dou, Yang Yi, Hongyi Qi, and Qinwan Huang analyzed the data and interpreted the results; Guojun Dou, Yang Yi, and Hongyi Qi developed the manuscript; Dejun Liu, Jianyou Shi, Guanrong Li, and $\mathrm{Na}$ Yang supervised the work and proofread the manuscript. All authors approved the manuscript. Yang Yi and Guojun Dou have contributed equally to this work.

\section{Acknowledgments}

This work was supported by the NSFC Projects (no. 81373903; no. 81202946), Chongqing Project of Science and Technology Talent Cultivation (cstc2013kjrc-qnrc1002), Chongqing Research Program of Basic Research and Frontier Technology (no. cstc2017jcyjAX0299), and Capacity Building Project for the Sustainable Utilization of Valuable Traditional Chinese Medicine Resources (no. 2060302).

\section{References}

[1] J. B. Harborne, "Role of Secondary Metabolites in Chemical Defence Mechanisms in Plants," in Ciba Foundation Symposium 154 - Bioactive Compounds from Plants, Novartis Foundation Symposia, pp. 126-139, John Wiley \& Sons, Ltd., Chichester, UK, 2007.

[2] S. Miresmailli and M. B. Isman, "Botanical insecticides inspired by plant-herbivore chemical interactions," Trends in Plant Science, vol. 19, no. 1, pp. 29-35, 2014.

[3] T. G. Son, S. Camandola, and M. P. Mattson, "Hormetic dietary phytochemicals," NeuroMolecular Medicine, vol. 10, no. 4, pp. 236-246, 2008.

[4] V. Calabrese, C. Cornelius, A. T. Dinkova-Kostova et al., "Cellular stress responses, hormetic phytochemicals and vitagenes in aging and longevity," Biochimica et Biophysica Acta, vol. 1822, no. 5, pp. 753-783, 2012.

[5] V. Murugaiyah and M. P. Mattson, "Neurohormetic phytochemicals: an evolutionary-bioenergetic perspective," Neurochemistry International, vol. 89, pp. 271-280, 2015.

[6] M. P. Mattson, "Hormesis defined," Ageing Research Reviews, vol. 7, no. 1, pp. 1-7, 2008.

[7] D. O. Kennedy and E. L. Wightman, "Herbal extracts and phytochemicals: plant secondary metabolites and the enhancement of human brain function," Advances in Nutrition, vol. 2, no. 1, pp. 32-50, 2011.

[8] D. Kultz, "Molecular and evolutionary basis of the cellular stress response," Annual Review of Physiology, vol. 67, pp. 225-257, 2005.

[9] Z. Zhao, P. Guo, and E. Brand, "The formation of daodi medicinal materials," Journal of Ethnopharmacology, vol. 140, no. 3, pp. 476-481, 2012.

[10] R. Yan, S. L. Li, H. S. Chung, Y. Tam, and G. Lin, "Simultaneous quantification of 12 bioactive components of Ligusticum chuanxiong Hort. by high-performance liquid chromatography," Journal of Pharmaceutical and Biomedical Analysis, vol. 37, no. 1, pp. 87-95, 2005.

[11] X. Kuang, Y. Yao, J. R. Du, Y. X. Liu, C. Y. Wang, and Z. M. Qian, "Neuroprotective role of Z-ligustilide against forebrain ischemic injury in ICR mice," Brain Research, vol. 1102, no. 1, pp. 145-153, 2006.

[12] X. Kuang, J.-R. Du, Y.-X. Liu, G.-Y. Zhang, and H.-Y. Peng, "Postischemic administration of Z-Ligustilide ameliorates cognitive dysfunction and brain damage induced by permanent 
forebrain ischemia in rats," Pharmacology Biochemistry \& Behavior, vol. 88, no. 3, pp. 213-221, 2008.

[13] X.-M. Wu, Z.-M. Qian, L. Zhu et al., "Neuroprotective effect of ligustilide against ischaemia-reperfusion injury via upregulation of erythropoietin and down-regulation of RTP801," British Journal of Pharmacology, vol. 164, no. 2, pp. 332-343, 2011.

[14] J. Yu, Z. Jiang, L. Ning et al., "Protective HSP70 induction by Z-ligustilide against oxygen-glucose deprivation injury via activation of the MAPK pathway but not of HSF1," Biological \& Pharmaceutical Bulletin, vol. 38, no. 10, pp. 1564-1572, 2015.

[15] H. Qi, Y. Han, and J. Rong, "Potential roles of PI3K/Akt and Nrf2-Keap1 pathways in regulating hormesis of Z-ligustilide in PC12 cells against oxygen and glucose deprivation," Neuropharmacology, vol. 62, no. 4, pp. 1659-1670, 2012.

[16] J. Li, J. Yu, H. Ma et al., "Intranasal Pretreatment with ZLigustilide, the Main Volatile Component of Rhizoma Chuanxiong, Confers Prophylaxis against Cerebral Ischemia via Nrf2 and HSP70 Signaling Pathways," Journal of Agricultural and Food Chemistry, vol. 65, no. 8, pp. 1533-1542, 2017.

[17] M. Miyazawa, T. Tsukamoto, J. Anzai, and Y. Ishikawa, "Insecticidal effect of phthalides and furanocoumarins from Angelica acutiloba against Drosophila melanogaster," Journal of Agricultural and Food Chemistry, vol. 52, no. 14, pp. 4401-4405, 2004.

[18] T. Tsukamoto, Y. Ishikawa, and M. Miyazawa, "Larvicidal and adulticidal activity of alkylphthalide derivatives from rhizome of Cnidium officinale against Drosophila melanogaster," Journal of Agricultural and Food Chemistry, vol. 53, no. 14, pp. 55495553, 2005.

[19] D. E. Wedge, J. A. Klun, N. Tabanca et al., "Bioactivity-guided fractionation and GC/MS fingerprinting of Angelica sinensis and Angelica archangelica root components for antifungal and mosquito deterrent activity," Journal of Agricultural and Food Chemistry, vol. 57, no. 2, pp. 464-470, 2009.

[20] S.-H. Chae, S.-I. Kim, S.-H. Yeon, S.-W. Lee, and Y.-J. Ahn, "Adulticidal activity of phthalides identified in cnidium officinale rhizome to B- and Q-biotypes of bemisia tabaci," Journal of Agricultural and Food Chemistry, vol. 59, no. 15, pp. 8193-8198, 2011.

[21] Q. Hou, "The correlations of the different host plants with preference level, life duration and survival rate of Spodoptera litura Fabricius," Chinese Journal of Eco-agriculture, 2004.

[22] J. Yu, Y. Li, M. Xiang et al., "Molecular cloning and characterization of $\alpha$-amylase/subtilisin inhibitor from rhizome of Ligusticum chuanxiong," Biotechnology Letters, vol. 39, no. 1, pp. 141-148, 2017.

[23] J. Liu, S. Zheng, L. Liu, L. Li, and Q. Feng, "Protein profiles of the midgut of spodoptera litura larvae at the sixth instar feeding stage by shotgun ESI-MS approach," Journal of Proteome Research, vol. 9, no. 5, pp. 2117-2147, 2010.

[24] K. Baskar and S. Ignacimuthu, "Antifeedant, larvicidal and growth inhibitory effects of ononitol monohydrate isolated from Cassia tora L. against Helicoverpa armigera (Hub.) and Spodoptera litura (Fab.) (Lepidoptera: Noctuidae)," Chemosphere, vol. 88, no. 4, pp. 384-388, 2012.

[25] Q. J. Chen, J. Li, and H. Pang, "A simple artificial diet for mass rearing of some noctuid species," Entomological Knowledge, vol. 6, pp. 325-327, 2000.

[26] W. S. Abbott, "A method of computing the effectiveness of an insecticide," Journal of the American Mosquito Control Association, vol. 3, no. 2, pp. 302-303, 1987.
[27] R. Wang, Y. Sun, X. Liang et al., "Effects of six plant secondary metabolites on activities of detoxification enzymes in Spodoptera litura," Shengtai Xuebao/ Acta Ecologica Sinica, vol. 32, no. 16, pp. 5191-5198, 2012.

[28] Y. Huang, Z. Xu, X. Lin, Q. Feng, and S. Zheng, "Structure and expression of glutathione $S$-transferase genes from the midgut of the Common cutworm, Spodoptera litura (Noctuidae) and their response to xenobiotic compounds and bacteria," Journal of Insect Physiology, vol. 57, no. 7, pp. 1033-1044, 2011.

[29] H. Qi, L. Li, and H. Ma, "Cellular stress response mechanisms as therapeutic targets of ginsenosides," Medicinal Research Reviews, vol. 38, no. 2, pp. 625-654, 2018.

[30] G. A. Howe and G. Jander, "Plant immunity to insect herbivores," Annual Review of Plant Biology, vol. 59, pp. 41-66, 2008.

[31] A. Mithöfer and W. Boland, "Plant defense against herbivores: Chemical aspects," Annual Review of Plant Biology, vol. 63, pp. 431-450, 2012.

[32] H. Zeng, G. Ni, L. He et al., "Law of Occurrence and the Damage of Main Diseases and Pests in Ligusticum chuanxiong Hort Field," Southwest China Journal of Agricultural Sciences, vol. 22, no. 1, pp. 99-101, 2009.

[33] R. Nakata, Y. Kimura, K. Aoki et al., "Inducible De Novo Biosynthesis of Isoflavonoids in Soybean Leaves by Spodoptera litura Derived Elicitors: Tracer Techniques Aided by High Resolution LCMS," Journal of Chemical Ecology, vol. 42, no. 12, pp. 1226-1236, 2016.

[34] S. Murakami, R. Nakata, T. Aboshi et al., "Insect-Induced Daidzein, Formononetin and Their Conjugates in Soybean Leaves," Metabolites, vol. 4, no. 3, pp. 532-546, 2014.

[35] S. Textor and J. Gershenzon, "Herbivore induction of the glucosinolate-myrosinase defense system: Major trends, biochemical bases and ecological significance," Phytochemistry Reviews, vol. 8, no. 1, pp. 149-170, 2009. 


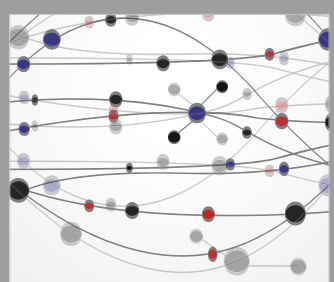

The Scientific World Journal
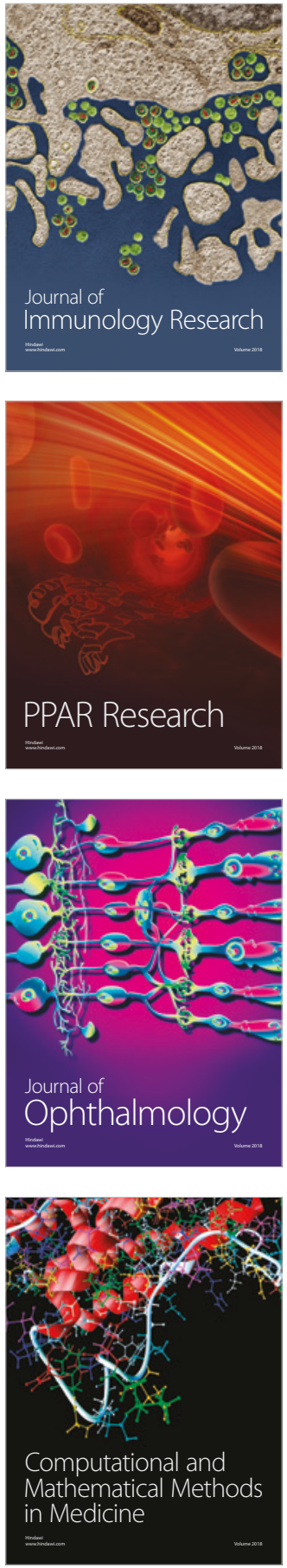

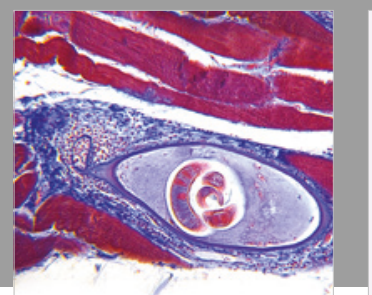

Gastroenterology Research and Practice

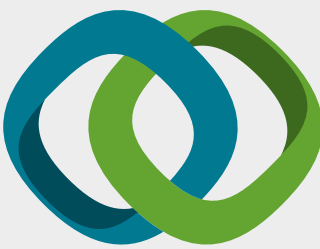

\section{Hindawi}

Submit your manuscripts at

www.hindawi.com
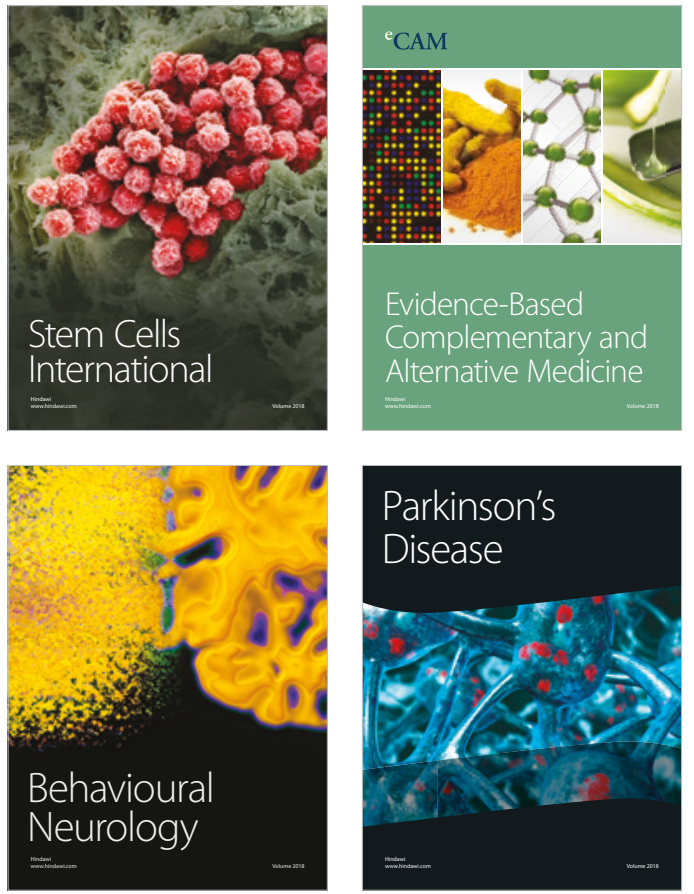

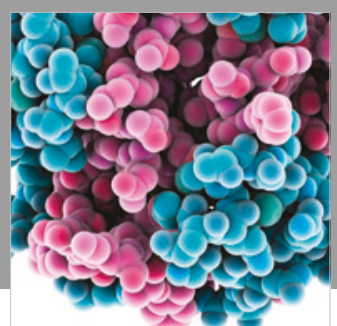

ournal of

Diabetes Research

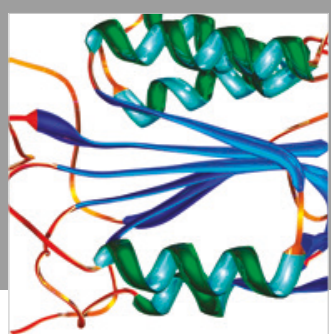

Disease Markers
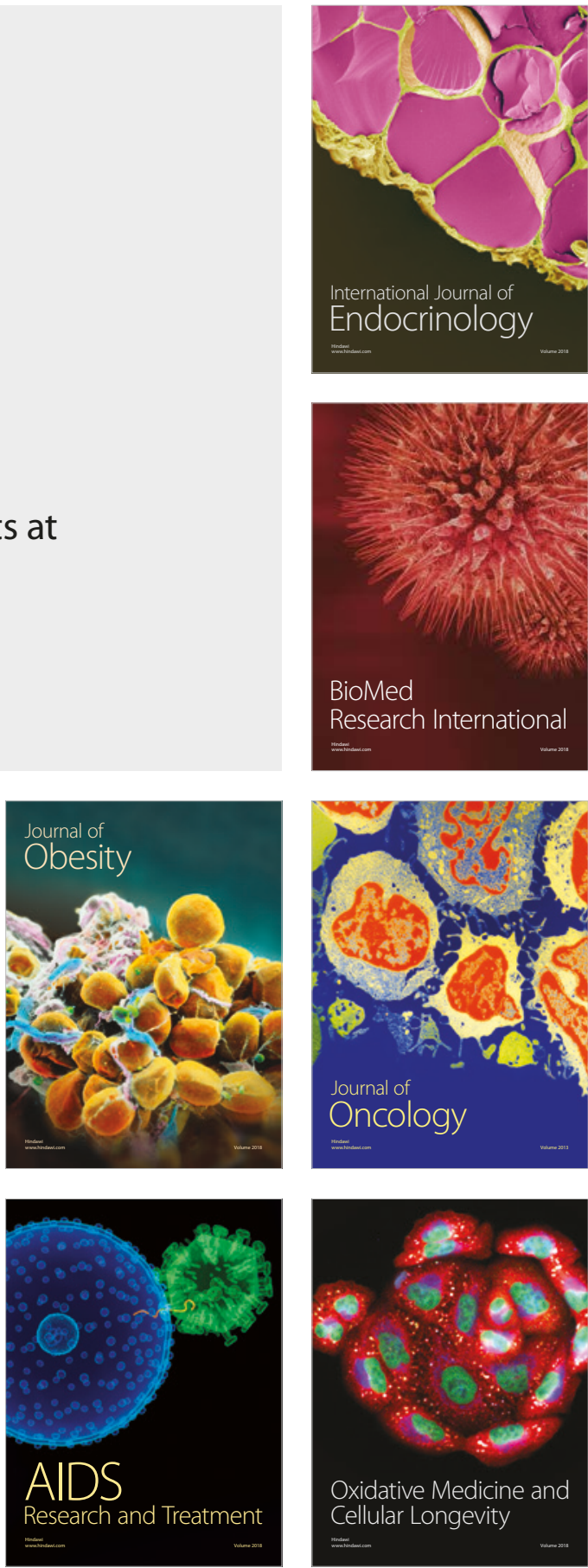